\title{
UNA PROPUESTA DIDÁCTICA PARA LA IMPLEMENTACIÓN DE LA ATENUACIÓN EN ESPAÑOL PARA LOS NEGOCIOS*
}

\section{A DIDACTIC PROPOSAL FOR THE IMPLEMENTATION OF MITIGATION IN SPANISH FOR BUSINESS}

\author{
Amanda Pulido Morera \\ Universitat de València \\ amanda.pulido94@gmail.com \\ Jorge Martí Contreras \\ Universitat Jaume I \\ Grupo de investigación Es.Vag.Atenuación / Val.Es.Co (Universitat de València) \\ / Sociolingüística (Universitat Jaume I) \\ jmarti@uji.es
}

Recibido: $20 / 12 / 2016$

Aceptado: 28/04/2017

\begin{abstract}
Resumen
El objetivo de este estudio es presentar una propuesta didáctica sobre la enseñanza de la atenuación en el ámbito de Español para los negocios, en Español como Lengua Extranjera. El diseño de esta propuesta tiene dos pilares: el análisis de materiales didácticos de E/LE de acuerdo con el Marco Común Europeo de Referencia en el nivel B1 y también se elabora un estudio de manuales. El resultado del análisis nos sirve para ver la frecuencia con la que
\end{abstract}

\begin{abstract}
The aim of this study is to offer a didactic proposal for teaching mitigation in Spanish $\mathrm{Bu}-$ siness, in Spanish as a Second Language. The design of this proposal has been based on the analysis of Spanish materials for specific purposes in accordance with the Common European Framework of Reference for Languages at the B1 level and in the analysis of books for Spanish Business. The result of this research has the aim of studying the frequency that mitigation is
\end{abstract}

\footnotetext{
* Este trabajo se enmarca en el proyecto La atenuación pragmática en su variación genérica: géneros discursivos escritos y orales en el español de España y América (referencia FFI2016-75249-P), financiado por el Ministerio de Economía, Industria y Competitividad y cuyas investigadoras principales son la Dra. Marta Albelda Marco y la Dra. María Estellés Arguedas.
}

Para citar este artículo / To cite this article: Pulido Morera, Amanda y Martí Contreras, Jorge (2018): Una propuesta didáctica para la implementación de la atenuación en español para los negocios. García Ramón, Amparo y Soler Bonafont, María Amparo (Eds.): ELUA: Estudios de atenuación en el discurso, Anexo IV, págs. 209-302.

Enlace / Link: http://dx.doi.org/10.14198/ELUA2018.Anexo4.16 
se lleva al aula el fenómeno de la atenuación. Después, se propone una secuencia didáctica implementada en la Universitat de València.

PALABRAS CLAVE: E/LE, EFE, atenuación, mitigación, estrategia pragmático-lingüística. studied during the lessons. Finally, we propose a teaching unit implemented at the University of Valencia (Spain).

KEY WORDS: E/LE, EFE, mitigation, pragmatic-linguistic strategy.

\section{INTRODUCCIÓN}

Dentro del ámbito de la didáctica de segundas lenguas apenas se aborda en el aula el fenómeno lingüístico de la atenuación ya que los cursos de Español como Lengua Extranjera (E/LE) están dentro de una programación institucional que da poco margen de maniobra al profesor y se ve obligado a llevar al aula, en muchas ocasiones, solo los contenidos gramaticales y léxicos mínimos que sugieren tanto el Marco Común Europeo de Referencia (MCER) como el Plan Curricular del Instituto Cervantes (PCIC). Por ese motivo, surgió el proyecto de analizar libros de Español para Fines Específicos (E/FE) como es el Español para los negocios, en el que resulta un tema esencial a la hora de abordar una negociación, ya que se debe analizar la mejor forma de hacer llegar el mensaje al interlocutor para conseguir llegar a un acuerdo, expresar su opinión sobre un tema, o para poder cerrar un pacto. Después de analizar los rasgos atenuantes de diferentes manuales que se encuentran en el mercado editorial, se diseña e implementa una unidad didáctica en la que se pone especial atención a la enseñanza de la atenuación.

\section{ANÁLISIS DE MATERIALES}

La primera parte de nuestro trabajo se centra en el análisis de un manual de Español para fines específicos, en concreto, Español para los negocios. El manual seleccionado es Socios 2, curso de español orientado al mundo del trabajo. El análisis de este manual se realiza en varias fases: en primer lugar, se ha realizado un análisis exhaustivo de la estructura de cada una de las unidades, tareas y actividades; y, en segundo lugar se han elegido tres unidades didácticas para determinar cómo se han diseñado tanto los textos escritos como orales. En tercer lugar, se han observado los aspectos de la atenuación de forma implícita. Y por último, se ha atendido a las actividades donde la atenuación se presenta explícitamente.

En cuanto a la temporalización de nuestro proceso de investigación, se ha realizado en dos partes. En primer lugar, hemos recogido todos los textos, tanto escritos como orales de las unidades seleccionadas en busca de rasgos atenuantes. En segundo lugar, hemos realizado una búsqueda con las actividades que presentan atenuación de forma implícita - no hemos encontrado atenuación explícita-.

Con este estudio, pretendemos descubrir la frecuencia de uso de la atenuación en manuales de E/FE. Asimismo, queremos saber cómo se lleva al aula y si hay una explotación suficiente de este aspecto pragmático y si se podría desarrollar de otra manera en la que se potencie más. El resultado de dicho análisis muestra la inclusión de una gran cantidad de rasgos atenuadores, pero que se abordan de forma indirecta y sin poner el foco de atención en ella. De modo que solo aparece de forma implícita y no se trabaja a posteriori; 
esto demuestra que no se pone el foco en la forma de atenuar, ya que aparece de forma incidental. Se muestra aquí una parte del corpus que fue extraído:

\begin{tabular}{|c|c|c|c|c|c|}
\hline Ejemplo & Página & Localización & Competencia & Procedimiento & Función \\
\hline $\begin{array}{l}\text { En esta unidad vamos a } \\
\text { elaborar el organigrama de } \\
\text { una empresa y a crear el } \\
\text { perfil de los responsables } \\
\text { de cada departamento }\end{array}$ & 10 & $\begin{array}{l}\text { Introducción } \\
\text { al tema }\end{array}$ & $\begin{array}{l}\text { Comprensión } \\
\text { lectora }\end{array}$ & $\begin{array}{l}\text { Nosotros } \\
\text { inclusivo }\end{array}$ & $\begin{array}{l}\text { Salvaguardad } \\
\text { el yo del tú }\end{array}$ \\
\hline $\begin{array}{l}\text { Para ello vamos a } \\
\text { aprender }\end{array}$ & 10 & $\begin{array}{l}\text { Introducción } \\
\text { al tema }\end{array}$ & $\begin{array}{c}\text { Comprensión } \\
\text { lectora }\end{array}$ & $\begin{array}{l}\text { Nosotros } \\
\text { inclusivo }\end{array}$ & $\begin{array}{l}\text { Salvaguardad } \\
\text { el yo del tú }\end{array}$ \\
\hline $\begin{array}{c}\text { Hoy es el primer día } \\
\text { de clase: preséntate a } \\
\text { tu compañero. Si ya os } \\
\text { conocéis, podéis comentar } \\
\text { qué habéis hecho estas } \\
\text { vacaciones. }\end{array}$ & 11 & $\begin{array}{l}\text { Descripción } \\
\text { de la } \\
\text { actividad }\end{array}$ & $\begin{array}{l}\text { Interacción } \\
\text { social }\end{array}$ & $\begin{array}{c}\text { Desplazamiento } \\
\text { de la } \\
\text { perspectiva } \\
\text { temporal }\end{array}$ & $\begin{array}{l}\text { Autoprotección } \\
\text { con imagen }\end{array}$ \\
\hline $\begin{array}{c}\text { Yo creo que estudias } \\
\text { español para conseguir un } \\
\text { buen trabajo }\end{array}$ & 11 & $\begin{array}{c}\text { Cuerpo de la } \\
\text { actividad }\end{array}$ & $\begin{array}{l}\text { Interacción } \\
\text { social }\end{array}$ & $\begin{array}{c}\text { Verbos } \\
\text { performativos }\end{array}$ & $\begin{array}{c}\text { Autoprotección } \\
\text { con imagen }\end{array}$ \\
\hline $\begin{array}{l}\text { ¿Qué creéis que hace cada } \\
\text { departamento? Coméntalo } \\
\text { con tu compañero }\end{array}$ & 12 & $\begin{array}{l}\text { Descripción } \\
\text { de la } \\
\text { actividad }\end{array}$ & $\begin{array}{l}\text { Interacción } \\
\text { social }\end{array}$ & $\begin{array}{c}\text { Verbos } \\
\text { performativos }\end{array}$ & $\begin{array}{l}\text { Autoprotección } \\
\text { con imagen }\end{array}$ \\
\hline $\begin{array}{c}\text { En el Departamento de } \\
\text { Contabilidad llevan las } \\
\text { cuentas de la empresa, } \\
\text { ¿no? }\end{array}$ & 12 & $\begin{array}{c}\text { Cuerpo de la } \\
\text { actividad }\end{array}$ & $\begin{array}{l}\text { Interacción } \\
\text { social }\end{array}$ & $\begin{array}{l}\text { Incertidumbre } \\
\text { o ignorancia }\end{array}$ & $\begin{array}{l}\text { Autoprotección } \\
\text { sin imagen }\end{array}$ \\
\hline $\begin{array}{l}\text { Hoy estoy un poco } \\
\text { cansado porque no he } \\
\text { dormido bien }\end{array}$ & 13 & $\begin{array}{c}\text { Cuerpo de la } \\
\text { actividad }\end{array}$ & $\begin{array}{l}\text { Interacción } \\
\text { social }\end{array}$ & $\begin{array}{c}\text { Un poco }+ \\
\text { adjetivo/ } \\
\text { adverbio }\end{array}$ & $\begin{array}{l}\text { Autoprotección } \\
\text { sin imagen }\end{array}$ \\
\hline $\begin{array}{l}\text { Elisa Valencia, experta } \\
\text { en Recursos Humanos, } \\
\text { ha sido entrevistada por } \\
\text { la revista Trabajo. Según } \\
\text { ella, ¿por qué es difícil } \\
\text { encontrar trabajo? }\end{array}$ & 15 & $\begin{array}{l}\text { Descripción } \\
\text { de la } \\
\text { actividad }\end{array}$ & $\begin{array}{l}\text { Comprensión } \\
\text { lectora }\end{array}$ & $\begin{array}{c}\text { Incertidumbre } \\
\text { o ignorancia }\end{array}$ & $\begin{array}{l}\text { Salvaguardad } \\
\text { el yo del tú }\end{array}$ \\
\hline $\begin{array}{c}\text { ¿Piensa usted que } \\
\text { hay aspectos que los } \\
\text { candidatos valoran mucho } \\
\text { y que las empresas, sin } \\
\text { embargo, valoran poco? }\end{array}$ & 15 & $\begin{array}{c}\text { Cuerpo de la } \\
\text { actividad }\end{array}$ & $\begin{array}{l}\text { Comprensión } \\
\text { lectora }\end{array}$ & Usted & $\begin{array}{l}\text { Salvaguardad } \\
\text { el yo del tú }\end{array}$ \\
\hline $\begin{array}{l}\text { A mí me parece que las } \\
\text { empresas buscan gente } \\
\text { con experiencia y que es } \\
\text { difícil tener un primer } \\
\text { trabajo }\end{array}$ & 15 & $\begin{array}{l}\text { Cuerpo de la } \\
\text { actividad }\end{array}$ & $\begin{array}{l}\text { Interacción } \\
\text { social }\end{array}$ & $\begin{array}{c}\text { Verbos } \\
\text { performativos }\end{array}$ & $\begin{array}{l}\text { Autoprotección } \\
\text { con imagen }\end{array}$ \\
\hline
\end{tabular}




\begin{tabular}{|c|c|c|c|c|c|}
\hline Ejemplo & Página & Localización & Competencia & Procedimiento & Función \\
\hline $\begin{array}{c}\text { En esta unidad vamos a } \\
\text { elaborar el organigrama de } \\
\text { una empresa y a crear el } \\
\text { perfil de los responsables } \\
\text { de cada departamento }\end{array}$ & 10 & $\begin{array}{c}\text { Introducción } \\
\text { al tema }\end{array}$ & $\begin{array}{c}\text { Comprensión } \\
\text { lectora }\end{array}$ & $\begin{array}{c}\text { Nosotros } \\
\text { inclusivo }\end{array}$ & $\begin{array}{c}\text { Salvaguardad } \\
\text { el yo del tú }\end{array}$ \\
\hline $\begin{array}{c}\text { Para ello vamos a } \\
\text { aprender }\end{array}$ & 10 & $\begin{array}{c}\text { Introducción } \\
\text { al tema }\end{array}$ & $\begin{array}{c}\text { Comprensión } \\
\text { lectora }\end{array}$ & $\begin{array}{c}\text { Nosotros } \\
\text { inclusivo }\end{array}$ & $\begin{array}{c}\text { Salvaguardad } \\
\text { el yo del tú }\end{array}$ \\
\hline $\begin{array}{c}\text { Hoy es el primer día } \\
\text { de clase: preséntate a } \\
\text { tu compañero. Si ya os } \\
\text { conocéis, podéis comentar } \\
\text { qué habéis hecho estas } \\
\text { vacaciones. }\end{array}$ & 11 & $\begin{array}{c}\text { Descripción } \\
\text { de la } \\
\text { actividad }\end{array}$ & $\begin{array}{c}\text { Interacción } \\
\text { social }\end{array}$ & $\begin{array}{c}\text { Desplazamiento } \\
\text { de la } \\
\text { perspectiva } \\
\text { temporal }\end{array}$ & $\begin{array}{c}\text { Autoprotección } \\
\text { con imagen }\end{array}$ \\
\hline $\begin{array}{c}\text { Yo creo que estudias } \\
\text { español para conseguir un } \\
\text { buen trabajo }\end{array}$ & 11 & $\begin{array}{c}\text { Cuerpo de la } \\
\text { actividad }\end{array}$ & $\begin{array}{c}\text { Interacción } \\
\text { social }\end{array}$ & verformativos \\
parbos & $\begin{array}{c}\text { Autoprotección } \\
\text { con imagen }\end{array}$ \\
\hline
\end{tabular}

Tabla 1. Ejemplos de elementos atenuadores en el texto del manual de E/FE.

\begin{tabular}{|c|c|c|c|c|c|}
\hline Ejemplo & $\begin{array}{c}\text { Pista } \\
\text { de } \\
\text { audio }\end{array}$ & Localización & Competencia & Procedimiento & Función \\
\hline No lo creo... $(\rightarrow)$ & Pista 1 & $\begin{array}{l}\text { Cuerpo de la } \\
\text { actividad }\end{array}$ & $\begin{array}{l}\text { Comprensión } \\
\text { oral }\end{array}$ & $\begin{array}{c}\text { Verbos } \\
\text { performativos }\end{array}$ & $\begin{array}{l}\text { Autoprotección } \\
\text { con imagen }\end{array}$ \\
\hline $\begin{array}{l}\text { Uf, pues la verdad que no } \\
\text { sé, estoy cansado }\end{array}$ & Pista 2 & $\begin{array}{l}\text { Cuerpo de la } \\
\text { actividad }\end{array}$ & $\begin{array}{l}\text { Comprensión } \\
\text { oral }\end{array}$ & $\begin{array}{l}\text { Incertidumbre o } \\
\text { ignorancia }\end{array}$ & $\begin{array}{l}\text { Autoprotección } \\
\text { con imagen }\end{array}$ \\
\hline $\begin{array}{c}\text { No sé, no me encuentro } \\
\text { muy bien }\end{array}$ & Pista 2 & $\begin{array}{l}\text { Cuerpo de la } \\
\text { actividad }\end{array}$ & $\begin{array}{l}\text { Comprensión } \\
\text { oral }\end{array}$ & $\begin{array}{l}\text { Incertidumbre o } \\
\text { ignorancia }\end{array}$ & $\begin{array}{l}\text { Autoprotección } \\
\text { con imagen }\end{array}$ \\
\hline $\begin{array}{c}\text { Uf, me parece que tú estás } \\
\text { enfermo }\end{array}$ & Pista 2 & $\begin{array}{l}\text { Cuerpo de la } \\
\text { actividad }\end{array}$ & $\begin{array}{l}\text { Comprensión } \\
\text { oral }\end{array}$ & $\begin{array}{c}\text { Verbos } \\
\text { performativos }\end{array}$ & $\begin{array}{l}\text { Autoprotección } \\
\text { con imagen }\end{array}$ \\
\hline $\begin{array}{l}\text { Creo que somos muchos } \\
\text { candidatos }\end{array}$ & Pista 3 & $\begin{array}{l}\text { Cuerpo de la } \\
\text { actividad }\end{array}$ & $\begin{array}{l}\text { Comprensión } \\
\text { oral }\end{array}$ & $\begin{array}{c}\text { Verbos } \\
\text { performativos }\end{array}$ & $\begin{array}{l}\text { Autoprotección } \\
\text { con imagen }\end{array}$ \\
\hline $\begin{array}{l}\text { Pues podrías irte un rato al } \\
\text { gimnasio, no sé }\end{array}$ & Pista 3 & $\begin{array}{l}\text { Cuerpo de la } \\
\text { actividad }\end{array}$ & $\begin{array}{l}\text { Comprensión } \\
\text { oral }\end{array}$ & $\begin{array}{c}\text { Desplazamiento } \\
\text { de la perspectiva } \\
\text { temporal }\end{array}$ & $\begin{array}{l}\text { Autoprotección } \\
\text { con imagen }\end{array}$ \\
\hline $\begin{array}{l}\text { Pues podrías irte un rato al } \\
\text { gimnasio, no sé }\end{array}$ & Pista 3 & $\begin{array}{l}\text { Cuerpo de la } \\
\text { actividad }\end{array}$ & $\begin{array}{l}\text { Comprensión } \\
\text { oral }\end{array}$ & $\begin{array}{l}\text { Incertidumbre o } \\
\text { ignorancia }\end{array}$ & $\begin{array}{l}\text { Autoprotección } \\
\text { con imagen }\end{array}$ \\
\hline $\begin{array}{l}\text { Mira, si quieres después } \\
\text { podemos comer juntos }\end{array}$ & Pista 3 & $\begin{array}{l}\text { Cuerpo de la } \\
\text { actividad }\end{array}$ & $\begin{array}{l}\text { Comprensión } \\
\text { oral }\end{array}$ & $\begin{array}{c}\text { Verbos } \\
\text { performativos }\end{array}$ & $\begin{array}{l}\text { Autoprotección } \\
\text { sin imagen }\end{array}$ \\
\hline $\begin{array}{c}\text { El que es un poco difícil } \\
\text { es Luis }\end{array}$ & Pista 5 & $\begin{array}{l}\text { Cuerpo de la } \\
\text { actividad }\end{array}$ & $\begin{array}{l}\text { Comprensión } \\
\text { oral }\end{array}$ & $\begin{array}{c}\text { Un poco }+ \\
\text { adjetivo/adverbio }\end{array}$ & $\begin{array}{l}\text { Autoprotección } \\
\text { sin imagen }\end{array}$ \\
\hline $\begin{array}{l}\text { Yo no tengo mucha } \\
\text { relación con él }\end{array}$ & Pista 5 & $\begin{array}{l}\text { Cuerpo de la } \\
\text { actividad }\end{array}$ & $\begin{array}{l}\text { Comprensión } \\
\text { oral }\end{array}$ & $\begin{array}{l}\text { No }+ \text { verbo }+ \\
\text { mucho/a }\end{array}$ & $\begin{array}{l}\text { Autoprotección } \\
\text { con imagen }\end{array}$ \\
\hline
\end{tabular}

Tabla 2. Ejemplos de elementos atenuadores en el texto del manual de E/FE. 
Explicamos a continuación los resultados obtenidos del vaciado de las tres unidades didácticas y sus respectivas pistas de audio. Los rasgos atenuadores que cuantitativamente son más frecuentes en el manual; son los siguientes:

- Uso del usted

- Verbo performativo: “Cree que deberíamos mandarle algún regalo?” (pista 88)

- El condicional: "Imagina que vas a dar un discurso de despedida. ¿Qué cosas harías en esta situación?” (pág. 132)

- Nosotros inclusivo: "En esta unidad vamos a redactar un informe sobre una feria. Para ello vamos a aprender [...]" (pág. 66)

- Incertidumbre o ignorancia: “Esto es el almacén, ¿no?” (pág. 67)

- Desplazamiento de la perspectiva temporal: «Hoy es el primer día de clase: preséntate a tu compañero. Si ya os conocéis, podéis comentar qué habéis hecho estas vacaciones» (pág. 11).

- Disculpas: "Es que no hemos podido mirarlo todavía, lo siento" (pista 88).

- Un poco + adjetivo/adverbio: "Hoy estoy un poco cansado porque no he dormido bien" (pág. 13)

- Sustantivo + bastante + adjetivo/adverbio: "Yo creo que tú eres un viajero bastante aventurero, ¿no?, porque [...]” (pág. 134)

A partir de estos datos nos damos cuenta de que hay un aumento progresivo de las actividades que de forma implícita trabajan la atenuación a medida que avanzamos en el temario.

\begin{tabular}{|c|c|c|}
\hline Unidad 1 & Unidad 6 & Unidad 12 \\
\hline 1 & 2 & 13 \\
\hline
\end{tabular}

Tabla 3. Elementos atenuadores trabajos de forma implícita, por unidades.

Este aumento significativo en el número de actividades, podríamos entenderlo en relación al nivel de los estudiantes. Es decir, el número de actividades que incluyen atenuación, aumenta a medida que los alumnos avanzan en el estudio del manual y por lo tanto, amplían su nivel en la LE. La atenuación, como otras estrategias pragmáticas necesita una base cognoscitiva sólida, por lo que no es casual que las primeras unidades se centren más en ofrecer léxico y gramática, y que, progresivamente, se ofrezcan estructuras atenuadoras más complicadas.

Debemos señalar que no hay actividades en las que la atenuación se explique de forma explícita. Si no aparece, es posible que los estudiantes no presten atención a la forma (Focus on Form) y por lo tanto, no adquieran ese conocimiento al no considerarlo relevante.

\section{DISEÑO E IMPLEMENTACIÓN DE UNA UNIDAD DIDÁCTICA}

\subsection{Descripción}

Los resultados obtenidos por el análisis de los materiales didácticos del mercado editorial nos lleva a la creación, diseño e implementación de una unidad didáctica con el objetivo 
de dar a conocer y trabajar la atenuación. Nuestro objetivo es que los alumnos aprendan qué es y para qué sirve, que conozcan su importancia en el ámbito de los negocios y que adquieran la habilidad de poder usarla para afrontar situaciones de la vida cotidiana en la que se debe utilizar este recurso pragmático.

Se ha elaborado una unidad didáctica de tres sesiones de dos horas, en cada una de las sesiones se trabajan unos aspectos distintos de la atenuación, por lo que cada sesión tiene unos objetivos gramaticales, léxicos y funcionales específicos. Los contenidos de la unidad didáctica están relacionados con los objetivos, y se han seleccionado tras el análisis del MCER y del Plan Curricular del Instituto Cervantes. Los objetivos que se deben cumplir son estos:

Entender la importancia de la atenuación, sobre todo en el ámbito de los negocios.

Adquirir expresiones lingüísticas necesarias para poder hacer un buen uso de la atenuación:

Estructuras internas a una oración (como: es un poco pesado)

Expresar acuerdo y desacuerdo

Dar la opinión

Dar consejos

Familiarizarse con expresiones atenuativas tanto de modo escrito como oral.

La implementación de la unidad didáctica se ha llevado a cabo en dos grupos (treinta y cinco personas en cada uno) de la Facultat d'Economia de la Universitat de València del segundo semestre del curso 2015/16, esto que permite observar dobles resultados y comprarlos. El nivel de los alumnos es un nivel umbral (B1). Hemos comprobado que en el PCIC en niveles inferiores al B1 no hay una presencia destacable de la atenuación. Es a partir del nivel B1, cuando podemos incluirla en los cursos, ya que los alumnos tienen una cierta madurez lingüística para ser capaces de asimilar la adquisición de esta estrategia pragmática.

En la última sesión, los estudiantes han realizado una autoevaluación. La autoevaluación es útil tanto para los alumnos como para el profesorado, ya que por una parte ellos conocen en primera persona si han asimilado o no lo estudiado en clase, y los docentes evalúan si los estudiantes han adquirido los procesos atenuadores de forma total o parcial y qué estructuras se necesita reforzar.

\subsection{Temporalización}

La unidad didáctica se ha dividido en tres sesiones en las que se han trabajo aspectos de la atenuación, así como expresiones y usos lingüísticos. El orden establecido ha sido de más fácil a más difícil, y se ha introducido en cada sesión algún elemento nuevo y se ha remitido a elementos anteriores.

En la primera sesión se presenta qué es la atenuación y porqué es importante estudiarla. Para ello, se explican a los estudiantes dos situaciones muy comunes donde es necesario atenuar. Además, se les comenta que esta estrategia pragmática también es muy necesaria para tener éxito en el ámbito de la negociación. Asimismo, se ha hecho uso de distintos temas para introducir la gramática. En esta primera sesión el tema que engloba la unidad es la publicidad y la televisión, por lo que se han utilizados dos vídeos publicitarios para mostrar casos muy diversos: en uno no se utiliza atenuación, en el segundo sí. 
A continuación, han visionado unos minutos de una entrevista en un programa de televisión en que la invitada se muestra enfadada con los presentadores, ya que se ha tomado muchas molestias para acudir al programa y piensa que el encuentro ha sido demasiado breve e infructuoso. Con esto, se pretende que los estudiantes adquieran material lingüístico necesario tanto para mostrar el enfado, como para calmar el enfado de otra persona. Para finalizar la sesión, a partir de un texto real obtenido del grupo de investigación Val.Es.Co. y adaptado al nivel de los alumnos, se han trabajado una serie de construcciones atenuantes internas de la oración.

En la segunda sesión se trabajan distintas funciones en las que la atenuación juega un papel importante. Estas funciones son: mostrar acuerdo y desacuerdo, por un lado; y, dar la opinión y hacer sugerencias, por otro. Los contenidos se han presentado a través del tema del cine, el cual guarda relación con la temática seguida en la primera sesión. Tanto en la publicidad como el cine se pretende vender un producto, hecho que abordaremos también en la tarea final.

Al final de esta sesión se realizará un juego de rol en el que los alumnos recibirán una tarjeta con una situación que deberán solucionar hablando con otro compañero. Para encontrar al compañero indicado se han seleccionado unas parejas de cine. En cada tarjeta aparece el nombre de un personaje, de modo que cada persona debe buscar a su otra pareja. Este juego nos servirá para repasar todas las estructuras estudiadas hasta el momento.

En la última sesión se ha llevado a cabo una tarea final, en la que por grupos han preparado la descripción de un producto novedoso, con todas sus características. Posteriormente, han intentado vender el producto al resto de la clase de la mejor forma posible. Para mantener activos a los estudiantes que no han realizado la presentación, el resto de grupos debía valorar el trabajo a través de una encuesta. Esta valoración se ha hecho en grupo, de manera que, de nuevo, deben emplear las funciones que hemos aprendido: dar la opinión, mostrar acuerdo y desacuerdo. Esta es una manera implícita de practicar la atenuación.

\begin{tabular}{|c|l|}
\hline \multirow{3}{*}{ Primera sesión } & $\begin{array}{l}\text { Introducción a la atenuación: descripción y uso. } \\
\text { Situaciones comunicativas con elementos atenuadores. } \\
\text { Atenuación en publicidad y televisión } \\
\text { Anuncio: "Permítame que insista" } \\
\text { Entrevista (no atenuada) a Mercedes Milá (programa "Vaya semanita") } \\
\text { Breve texto del grupo Val.Es.Co. con elementos atenuadores }\end{array}$ \\
\hline Segunda sesión & $\begin{array}{l}\text { Descripción de películas. Funciones de la atenuación: expresar acuerdo y } \\
\text { desacuerdo, dar la opinión, hacer sugerencias... } \\
\text { Opinión sobre algunas películas muy comerciales (mural). } \\
\text { Actividad final de revisión de los contenidos de las sesiones 1 y 2. }\end{array}$ \\
\hline Tercera sesión & $\begin{array}{l}\text { Descripción de un producto novedoso con la finalidad de venderlo a los } \\
\text { compañeros de clase. } \\
\text { Se deben emplear las técnicas de atenuación vistas } \\
\text { Autoevaluación }\end{array}$ \\
\hline
\end{tabular}

Tabla 4. Cronograma.

Para terminar con esta unidad didáctica, los alumnos han completado una autoevaluación, herramienta que permite observar el grado de adquisición de los contenidos en los alumnos. Se les entrega una rúbrica con preguntas y tres respuestas posibles: adquirido, adquirido parcialmente y no adquirido. 


\subsection{Características del alumnado}

Era el grupo de estudiantes internacional que procedía de países de Europa, Estados Unidos y Asia, por lo tanto era un grupo heterogéneo. La edad de los alumnos oscilaba entre los diecinueve y los veintiséis. Todos ellos estaban cursando el Grado de International Business en la Facultat d'Economia de la Universitat de València.

La unidad didáctica se ha implementado en la asignatura de Español para los negocios. Se trata de una unidad con unos fines específicos, por lo que el tema central son los negocios y, sobre todo, la publicidad. Con esto se pretende aproximar el fenómeno de la atenuación a los alumnos; por una parte, haciendo uso de unos temas relacionados con sus estudios vinculantes y, por otro lado, estos mismos temas nos parecen idóneos para desarrollar la atenuación, pues los fenómenos lingüísticos que explicamos aparecen en gran medida en la publicidad.

Se ha llevado a cabo el diseño de un material acorde a las particularidades de los estudiantes y que cumple con los propósitos, objetivos y contenidos establecidos en el syllabus del curso.

\subsection{Metodología y desarrollo de la unidad didáctica}

En la unidad didáctica se sigue un enfoque comunicativo por tareas porque de esta forma se asegura que el estudiante participe activamente en el proceso de adquisición de la lengua. Este enfoque incluye una tarea final en la que los alumnos desarrollan todos los contenidos trabajados durante la unidad didáctica.

La agrupación de los alumnos a lo largo de las sesiones ha sido distinta dependiendo del tipo de actividad: de forma individual (para actividades en las que se necesitaba que el estudiante buscara fenómenos atenuativos), parejas, tríos y grupos reducidos (para analizar documentos audiovisuales y propuesta de publicitación de un producto) y gran grupo -toda la clase- (para escuchar las propuestas de los otros grupos y realizar el lanzamiento de solo uno de los productos de todos los propuestos).

Trabajar con material audiovisual real en el aula de E/LE «It may be useful to expose learners to the pragmatic aspects of the target language» (Alcón y Safont 2008: 199). Es por eso, por lo que introducimos dos anuncios de poca duración, ya que son una muestra del uso de la atenuación en contextos reales.

Por otro lado, este fenómeno pragmático se trabaja en todo momento de forma explícita. Desde el primer momento se introduce a los alumnos en el tema central, se les explica qué es y se narran dos situaciones en las que necesitan hacer uso de esta estrategia. En el artículo de Alcón y Safont (2008: 199) podemos encontrar la importancia de trabajar este aspecto de forma explícita "As pointed out in various studies on pragmatic instructional intervention [...], explicit metapragmatic instruction seems to be more effective than implicit teaching" (2008: 199).

\subsection{Resultados de la implementación}

Tras el análisis de materiales de E/LE y después de la creación e implementación de la unidad didáctica, nos disponemos a valorar los resultados obtenidos. 


\subsubsection{Valoración de la metodología empleada}

Comentamos, en primer lugar, la metodología empleada y la secuenciación de las actividades. En segundo lugar, nos centramos en los resultados lingüísticos obtenidos a través de la autoevaluación y de la elaboración de actividades de expresión escrita. Finalmente, observamos los resultados obtenidos en las pruebas finales de la asignatura y comparamos estos resultados con las respuestas de la autoevaluación.

Partiendo de la observación directa en las clases durante la implementación, podemos presentar algunos resultados sobre el funcionamiento y la respuesta de ambos grupos durante el pilotaje:

a) No todos los alumnos presentaron una motivación hacia la explicación y el trabajo en clase. Algunos de ellos no mostraron el máximo grado de atención durante todas las sesiones. Eran dos grupos numerosos y no todos los alumnos tienen el mismo interés.

b) Además, la implementación se realizó a finales de su cuatrimestre, y algunos sentían más preocupación por los trabajos que debían realizar y el examen final, que por las clases.

c) Se mostraron motivados en los ejercicios donde predominaba la destreza de interacción oral. Se trabaja en pequeños grupos y los profesores participaban unos minutos en cada grupo para ver de qué manera se desarrollaba.

d) De esto, extraemos que los alumnos, en su mayoría, realizaban el ejercicio en español y preguntaban dudas de léxico, pronunciación, etc.

e) Asimismo, observamos una participación activa en la realización de actividades escritas.

f) Lo mismo sucede en la actividad en la que deben completar un mural en el que exponían su opinión personal sobre películas de distintos géneros.

h) Los vídeos trabajados en la primera sesión fueron demasiado complejos, y aunque se escucharon en tres ocasiones, deberíamos haberlos trabajado de otra forma para simplificar su complejidad, como, por ejemplo, entregar a los estudiantes la transcripción.

i) Por último, en la tarea final sobre el lanzamiento de un producto el alumnado, que trabajó en grupos de cuatro personas, mostró una gran originalidad a la hora de presentar los productos, inventó lemas, escenas teatrales breves muy divertidas que hicieron que el cierre de la implementación fuera el esperado. Algunos grupos crearon sus propios productos, otros eligieron de entre los ejemplos llevados a clase.

\subsubsection{Resultados de la implementación}

A continuación nos centramos en la observación y explicación de los resultados obtenidos en la autoevaluación. Resultados que responden a preguntas sobre la comprensión de los conceptos llevados al aula y sobre la adquisición de la atenuación. Para cada uno de los ítems tenían tres respuestas: adquirido, adquirido parcialmente y no adquirido. 
Aquí se muestra la tabla empleada y a continuación se realizan unos comentarios:

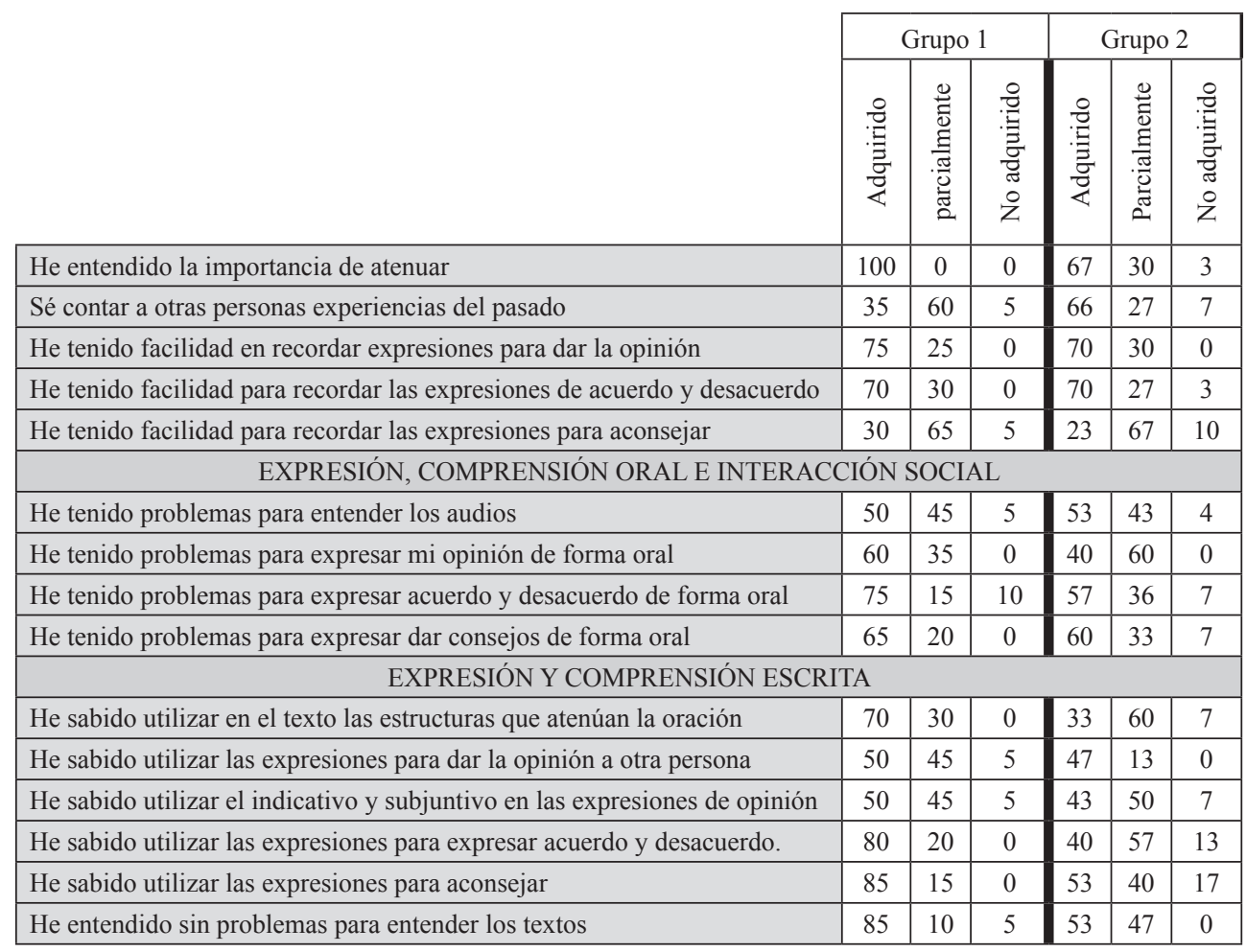

Tabla 5. Rúbrica sobre autoevaluación.

En rasgos generales podemos decir que:

a) La mayoría de los componentes de ambos grupos han respondido con bastante franqueza la autoevaluación. Sin embargo, encontramos algunas respuestas que a simple vista revelan la falta de sinceridad, después de ver el resultado final de la evaluación. En la respuesta a si han entendido qué es la atenuación en el Grupo 1, ya que el $100 \%$ de los alumnos han respondido que sí. Por el contrario, el resultado del Grupo 2 es de un $67 \%$.

b) Hemos tenido que prescindir de algunas encuestas por dos motivos: por una parte porque el alumno no ha acudido a las tres sesiones, y por tanto, no es una muestra válida; y por otra parte, porque el alumno ha respondido "adquirido" a toda la autoevaluación, y esto, obviamente no es una muestra fiable.

c) Muchas de las afirmaciones se han respondido de forma similar, sin embargo, otras respuestas distan bastante de un grupo al otro.

En primer lugar, veremos las diferencias entre los resultados de ambos grupos en el primer bloque. La primera desemejanza la encontramos ya en la primera pregunta, concretamente en el porcentaje de la respuesta de ambos grupos. Ocurre lo mismo en la 
segunda pregunta: en el Grupo 1, el $60 \%$ ha respondido parcialmente, mientras que en el Grupo 2, solo el $27 \%$ ha marcado esta misma respuesta, siendo una mayoría de un $70 \%$ los que consideran que han adquirido las estructuras para contar experiencias del pasado.

Las dos preguntas siguientes tienen unas respuestas muy similares: en ambos grupos, alrededor del $70 \%$ ha respondido "adquirido" en estas dos cuestiones. En la última pregunta del bloque también hay coincidencias entre los dos grupos; en este caso, la mayoría de los alumnos consideran que solo han adquirido parcialmente las estructuras para aconsejar, con un $65 \%$ (grupo 1) y un $67 \%$ (grupo 2).

Pasamos a comentar el segundo bloque de preguntas. En este caso, las cuatro preguntas presentan unos resultados semejantes: en la primera alrededor del $50 \%$ consideran que han tenido problemas para entender las actividades de comprensión auditiva (50\% el Grupo 1 y $53 \%$ el Grupo 2). Lo mismo ocurre con la última pregunta del bloque: el 65\% de alumnos del Grupo 1 y el 60 \% del Grupo 2 han tenido problemas para expresar consejos.

En las preguntas sobre los problemas que han tenido para expresar la opinión y manifestar acuerdo y desacuerdo (preguntas dos y tres del segundo bloque), observamos que hay diferencia entre las respuestas de ambos grupos. En el Grupo 1, el 60 \% y el $75 \%$ responden adquirido, por el contrario, en el Grupo 2, solamente el $40 \%$ y el $57 \%$ consideran adquirido. Como vemos, hay una diferencia del $20 \%$ aproximadamente entre un grupo y otro.

Para finalizar con la comparación entre las respuestas de ambos grupos, observamos que en el último bloque de preguntas hay una diferencia significativa entre los resultados obtenidos entre los Grupos 1 y 2 a simple vista vemos cómo el Grupo 2 muestra más dificultades en la utilización de estas estructuras gramaticales en las destrezas escritas. Mientras que en el Grupo 1, en todas las respuestas el $50 \%$ o más -llegando hasta un $80 \%$ - consideran que han sabido utilizar estas expresiones (adquirido), el Grupo 2 tiene porcentajes muy semejantes entre "adquirido totalmente" y "adquirido parcialmente".

Por último, debemos mencionar que los porcentajes en las respuestas de no adquirido son escasas. En la mayoría de los casos, el resultado es $0 \%$, y el porcentaje más alto que encontramos es de un $10 \%$ en la pregunta sobre la facilidad para recordar las expresiones para aconsejar en el Grupo 2, y otro $10 \%$ en el Grupo 1 en las estructuras para expresar acuerdo y desacuerdo de forma oral.

Seguidamente, observamos las preguntas con un mayor porcentaje en las respuestas de adquirido parcialmente o no adquirido. Prestaremos atención a estos aspectos ya que son los que presentan más dificultades en los estudiantes, y por lo tanto, los que no han sido adquiridos. Ambos grupos coinciden en la facilidad para recordar las expresiones para aconsejar. En general, el Grupo 2 presenta mucha más dificultad en la adquisición que el Grupo 1, sobre todo, en el último bloque de preguntas. Este aspecto se ve reflejado en la nota final ya que el grupo 1 ha obtenido $70,5 \%$ mientras que el grupo 2 solo ha sido capaz de lograr el $65 \%$.

Vemos, por lo tanto, que los resultados de la autoevaluación y los del examen final guardan relación. En la autoevaluación, el Grupo 1 consideraba "adquirido" la mayoría de las preguntas, sin embargo, el Grupo 2, respondía en ocasiones "adquirido parcialmente". Esta diferencia en la adquisición de las estructuras gramaticales, también se refleja en los resultados del examen. El material didáctico empleado es el mismo, por lo que deberíamos buscar la motivación de este menor rendimiento, por ejemplo, en la hora de la clase (grupo 1, de 15.30 a $17.30 \mathrm{~h}$; grupo 2, de 17.30 a 19.30 h) y podrían ya estar cansados; o tal vez, podría ser el menor nivel académico, en general, del grupo 2. 


\section{CONCLUSIÓN}

Podemos concluir que la atenuación es una estrategia pragmática tan necesaria como la gramática o el léxico en un manual de E/LE. Según Alcón y Safont (2008: 194) la enseñanza de la atenuación -o de cualquier aspecto pragmático- debe hacerse de una forma explícita. Sin embargo, tal y como hemos observado en el manual analizado, Socios 2, no presenta de forma explícita la atenuación, pese a seguir un enfoque comunicativo.

No obstante, sí que se encuentran actividades en la que de forma implícita se aborda este fenómeno lingüístico-pragmático. Así lo demuestra el estudio que hemos realizado sobre el manual Socios 2 y del que hemos reflejado una pequeña tabla del corpus extraído. En el corpus hemos analizado unidades didácticas que muestran cómo el manual incrementa en cada unidad el número de fenómenos atenuativos abordados.

Podemos advertir, partiendo de este hecho, que la atenuación debería incluirse atendiendo al nivel de lengua de los alumnos, motivo por el cual pensamos que los autores del manual deciden insertar esta estrategia pragmática al final del manual, pero seguramente el alumnado no tendrá el tiempo suficiente para asimilar estos conocimientos.

La segunda parte del presente trabajo ha consistido en la elaboración de una unidad didáctica centrada en la atenuación como principal objetivo y su posterior implementación en dos clases de Español para los negocios de la Facultat d'Economia. De esta puesta en práctica podemos destacar que no resultó complicado a los alumnos entender qué es la atenuación, cómo se emplea, cuándo y por qué motivo.

Por otra parte, podemos considerar que algunos de los contenidos de la unidad didáctica, podrían mejorarse. Tal es el caso de las actividades de la primera sesión, donde se visualizaban vídeos sobre entrevistas (a Mercedes Milá). El grado de dificultad era excesivo para el nivel B1, de manera que deberíamos simplificar dichas actividades, por ejemplo, añadiendo subtítulos o leyendo previamente el texto que escucharemos.

Por el contrario, hay algunas actividades con las que se ha trabajado bien y los alumnos han respondido positivamente. El tema del cine -en la segunda sesión- ha resultado entretenido y motivador por lo que se han podido llevar al aula estructuras gramaticales para dar la opinión, mostrar desacuerdo, hacer sugerencias, etc. gracias a este tema. Esto nos ha permitido trabajar de forma más cómoda que en la primera sesión.

Podemos considerar que una forma adecuada de empezar con la explicación del contenido, fue presentarles dos situaciones muy comunes en las que ellos mismos utilizan atenuación en su día a día. Asimismo, a lo largo de las tres sesiones, se intentó mostrar la utilidad de esta estrategia tanto en el ámbito formal como informal. Los alumnos pudieron practicarlo al final de la segunda sesión, en la que realizaron una actividad de interacción oral para practicar distintas situaciones habituales y debían resolver utilizando las estructuras estudiadas.

Finalmente, podríamos añadir que la gran mayoría de los alumnos, de ambas clases han considerado "adquiridos" o "parcialmente adquiridos" los elementos atenuativos llevadas al aula. Por lo tanto, podemos afirmar que la atenuación es una estrategia pragmática necesaria que debe explicarse en el aula, y como ha quedado demostrado en este estudio, es totalmente factible que el profesor la presente en clase tanto de forma implícita como de forma explícita. 


\section{Referencias bibliográficas}

Albelda, M. y A. Cestero (2011). "De nuevo, sobre los procedimientos de atenuación”, Español actual, 96, pp. 121-155.

Albelda, M. y A. Cestero (2012). "La atenuación lingüística como fenómeno variable". En Cestero, A. M., Molina, I., y F. Paredes (eds.). La lengua, lugar de encuentro. Actas XVI Congreso Internacional de la Alfal, pp. 1857-1866.

Albelda, M., Briz, A., Cestero, A. M., Kotwica, D. y C. Villalba (2014). "Ficha metodológica para el análisis pragmático de la atenuación en corpus discursivos del español. (ES.POR.ATENUACIÓN)", Oralia, 17, pp. 7-62.

Alcón, E. y M.P. Safont (2008). "Pragmatic awareness in second language acquisition", Encyclopedia of Language and Education, pp. 1948-1959.

Bartol, E. (2003). "La atenuación y la intensificación: herramientas para un mensaje adecuado", Tinkuy: Boletín de investigación y debate, pp. 47-55.

Bravo, D. (2005). "Categorías, tipologías y aplicaciones. Hacia una redefinición de la «cortesía comunicativa”. En D. Bravo (ed.). Estudios de la (des)cortesía en español. Categorías conceptuales y aplicaciones a corpora orales y escritos. Buenos Aires: Dunken, pp. 21-52.

Bravo, D. (2008). "Situación de habla, recursos comunicativos y factores lingüísticos en la interpretación de objetivos de cortesía". En Briz, A., Hidalgo, A., Albelda, M., Contreras, J. y Hernández Flores, N. (eds.). Cortesía y conversación: de lo escrito a lo oral. Valencia: Universitat de València, pp. 12-24.

Briz, A. (1995). "La atenuación en la conversación coloquial. Una categoría pragmática”, en Cortés, L. (ed.), El español coloquial. Actas del I Simposio sobre análisis del discurso oral. Universidad de Almería, pp. 103-122.

Briz, A.1996). El español coloquial: situación y uso. Madrid: Arco/Libros.

Briz, A. (1998). El español coloquial en la conversación. Esbozo de pragmagramática. Barcelona: Ariel.

Briz, A. (2006): "Atenuación y cortesía verbal en la conversación coloquial: su tratamiento en la clase de ELE". En Actas del programa de formación para profesorado de ELE. Munich: Instituto Cervantes, pp. 227-255: http://cvc.cervantes.es/ensenanza/biblioteca_ele/publicaciones_centros/PDF/ munich_2005-2006/02_briz.pdf (5-08-2017).

Briz, A. (2007). "Para un análisis semántico, pragmático y sociopragmático de la cortesía atenuadora en España y América", LEA, XIX, pp. 1-38.

Briz, A.y M. Albelda (2013). "Una propuesta teórica y metodológica para el análisis de la atenuación lingüística en español y portugués. La base de un proyecto común (ES.POR.ATENUACIÓN)", Onomazéin, 28, pp. 288-319.

Briz, A., Gómez, J.R., Martínez, M. a J. y Grupo Val.Es.Co. (1997). Pragmática y gramática del español hablado. Valencia: Libros Pórtico.

Caffi, C. (1999). “On mitigation”, Journal of pragmatics, 31, pp. 881-909

Consejo de Europa (2001). Marco común europeo de referencia para las lenguas: aprendizaje, enseñanza, evaluación. Madrid: MECD-Anaya

Cordulino da Silva, M. (2010). "Análisis de la presencia del registro coloquial en manuales de E/LE”, Trabajo de Final de Máster, Universitat de València.

Equipo pedagógico Nebrija (1998). Aportaciones de la pragmática a la enseñanza de español/LE. Fundación Antonio Nebrija.

Escandell Vidal, M.V. (2004). “Aportaciones de la pragmática”. En J. Sánchez Lobato e I. Santos Gargallo (dirs.). Enseñar español como segunda lengua o lengua extranjera. Vademécum para la formación de profesores. Madrid: SGEL, pp. 179-198.

Felix-Brasdefer, J.C. (2004). "La mitigación en el discurso oral de mexicanos y aprendices de español como lengua extranjera". En Briz, A. y D. Bravo (coords.). Pragmática sociocultural: estudios sobre el discurso de cortesía en español. Barcelona: Ariel, pp. 285-299. 
Felix-Brasdefer J. C. (2008). "Teaching Pragmatics in the Classroom: Instruction of Mitigation in Spanish as a Foreign Language", Hispania, Vol. 91, 2, pp. 479-494.

Fernández Loya, C. (2005). "Estrategias de intensificación y de atenuación en el español e italiano coloquiales", Actas del AISPI, núm. XXIII, pp. 188-200.

Hymes, D. (1971). On Communicative Competence. Filadelfia: Universidad de Pensilvania. Traducción al español en Llobera, M. (ed.) (1995), Competencia comunicativa. Documentos básicos en la enseñanza de lenguas extranjeras. Madrid: Edels, pp. 27-46.

Instituto Cervantes (2006). Plan Curricular del Instituto Cervantes. Niveles de referencia para el español. Madrid: Instituto Cervantes-Editorial Biblioteca Nueva.

Instituto Cervantes (2008). Diccionario de términos clave de ELE. Madrid: Instituto Cervantes-SGEL.

Martínez, L. y M.L. Sabater (2008). Socios 2, Barcelona, Difusión.

Medina, I. (2012). "Los elementos atenuadores para expresar desacuerdo en el discurso oral de estudiantes E/LE universitarios de nivel B1 en contexto de inmersión. Estudio cualitativo", Revista Nebrija de Lingüistica Aplicada a la Enseñanza de Lenguas, 11(6), pp. 104-143.

Medina, I. (2013). "Los elementos atenuadores para expresar desacuerdo en el discurso oral de estudiantes E/LE. Estudio cuantitativo", Marcoele: Revista de Didáctica, Vol. 16, pp. 1-31.

Meyer-Hermann, R. (1988). "Atenuación e intensificación (análisis pragmático de sus formas y funciones en el español hablado)". Anuario de Estudios Filológicos, 11, pp. 275- 290.

Robles Garrote, P. (2014). "Competencia plurilingüe y estrategias de atenuación en el cambio de código lingüístico de L1 a L2/L3”. En Nebrija Procedia, vol. 3, pp. 547-558. 